\title{
LV-VE-5
}

\section{Laparoscopic major (> 2 segments) hepatectomy in Greece}

\author{
loannis HATZARAS*, Nikolaos ARKADOPOULOS
}

Surgery, National and Kapodistrian University of Athens, Athens, Greece

Introduction: Despite gaining traction around the world, laparoscopic approach to major hepatectomy, defined as resection of at least two neighboring hepatic segments, has not been fully adopted in Greece. We sought to describe our growing experience.

Methods: Data on six patients who underwent major hepatic resection in the last year in two major urban hospitals.

Results: The predominant indication for hepatectomy in these patients were metastatic disease to the liver. Three patients had colorectal metastases, one patient had metastatic melanoma, and two patients had benign pathology. There were two males and four females, with an average age of 59 (range: 52-71 years). Four patients had a solitary lesion and two had two lesions. Four patients had received pre-hepatectomy disease-specific chemotherapy or immunotherapy. The median size of the lesion was $5 \mathrm{~cm}$ (range: $3.5-12 \mathrm{~cm}$ ). Two patients underwent a right hepatic lobectomy, one a left hepatic lobectomy together with a right colectomy, and three patients underwent right anterior sectorectomy. All operations were done purely laparoscopic except one, where a hand port was employed due to the size of the lesion. Median operative time was 190 minutes and median stay at the hospital was 2 nights. No patient required intensive care unit monitoring. There was no 30-day mortality.

Conclusions: Major laparoscopic hepatic resection can be accomplished safely in well selected patients in the current Greek health system. Patients who have a laparoscopic hepatectomy benefit from a short hospital stay, without jeopardizing the safety of the operation. 